\title{
Sustainability of cancer waiting times: the need to focus on pathways relevant to the cancer type
}

\author{
Christopher Slade ${ }^{1}$ Robert Talbot ${ }^{2}$
}

J R Soc Med 2007; 100:309-313

The NHS Cancer Plan $2000^{1}$ set specific targets for the time patients should wait before receiving treatment as a first step in reducing long waiting times and to improve outcomes for patients with cancer in the UK. The link between increased survival and shorter waiting times for breast cancer patients was suggested by Richards et $a .^{2}$ and has formed the basis of current waiting time targets. The first to be introduced was the 'two-week wait' (fast-track), requiring all patients suspected of having cancer to be referred within 24 hours and seen by a specialist within two weeks. Two further standards, to be complied with by December 2005, were imposed and are now collectively known as the 31- and 62-day standards. The 31-day standard requires all patients with cancer to be treated within 31 days of the decision to treat, and the 62-day standard requires all fast-track patients to be treated within 62 days of referral by a general practitioner (GP).

The Government acknowledged that 100\% compliance would not be possible and therefore a $98 \%$ target was set for the 31-day standard, with 95\% for the 62-day standard. Achievement of these targets, in Dorset, was accomplished in early 2006 and by most other cancer networks during 2006, but it became apparent that to make these gains 'sustainable' would require more work and investment. ${ }^{3}$ The work of the Cancer Service Collaborative 'Improvement Partnership' (CSCIP) and the Intensive Support Team (IST) during 2006 led to a greater understanding of the methology necessary for sustainability of compliance with the 62-day standard. This work has culminated in the identification of three main categories into which all Trusts could be placed: ${ }^{4}$

- Those achieving at or above 95\% through having effective pathways;

- Those achieving at or above 95\% through short-term methods;

- Those without effective systems who have yet to achieve.

\footnotetext{
${ }^{1}$ Service Improvement and Information Manager, and ${ }^{2}$ Medical Director, Dorset Cancer Network, Victoria House, Princes Road, Ferndown, Dorset BH22 9JR, UK

Correspondence to: Christopher Slade

Email: chris.slade@ferndown.nhs.uk
}

These three categories therefore identify those Trusts that have built sustainable processes for maintaining these standards. To aid the identification of sustainable pathways / processes the CSCIP and IST issued a sustainability graph which plotted 'percentage of all patients treated' against 'days waited for decision to treat'. Processes that had a high sustainability fell into the 'High Sustainability' zone, process that had a low sustainability fell into the 'Low Sustainability' zone, and similarly for medium sustainability and low-tomedium sustainability. The rationale behind this type of graph is that the sooner a patient reaches the decision to treat stage, the less likely they are to breach the 62-day standard.

Direct correspondence from the Cancer Action Team to Cancer Networks ${ }^{5}$ suggested that ' . . . the 62-day standard is most likely to be achieved and sustained when decision to treat is reached by day 42.'

Cancer waiting times for Dorset were downloaded from the Open Exeter Cancer Waiting Times database for each cancer type. The selection criterion was based on the first definitive treatment date (shared accountability search) option. The necessary data was then extracted from these downloads by a bespoke software programme ${ }^{6}$ written specifically for the Dorset Cancer Network; this compiled data by month, composite waiting times and the first definitive treatment for each patient for each cancer site.

Following a Strategic Health Authority request this data was used to produce a sustainability graph for all cancer patients in Dorset, which showed low-to-medium sustainability. It seemed highly unlikely that all our cancer pathways fitted into this sustainability band because we believed that the breast cancer pathway, for example, was very efficient. Therefore, each individual cancer site's sustainability graph was plotted with the hope that the nonsustainable pathways could be identified.

The sustainability graph for urology cancer patients showed a peculiar flat section moving between yellow (medium sustainability) and amber (low-to-medium sustainability) at a patient level of 50\%. This suggested either two parts to the pathway or groups of patients travelling at different speeds. It seemed illogical that patients should go through their pathway at distinctly different speeds and so this was investigated in more detail. We hoped to be able to identify those patients that were taking longer and possibly discover the reasons for this. 


\section{Bladder (C67) and Prostate (C61)}

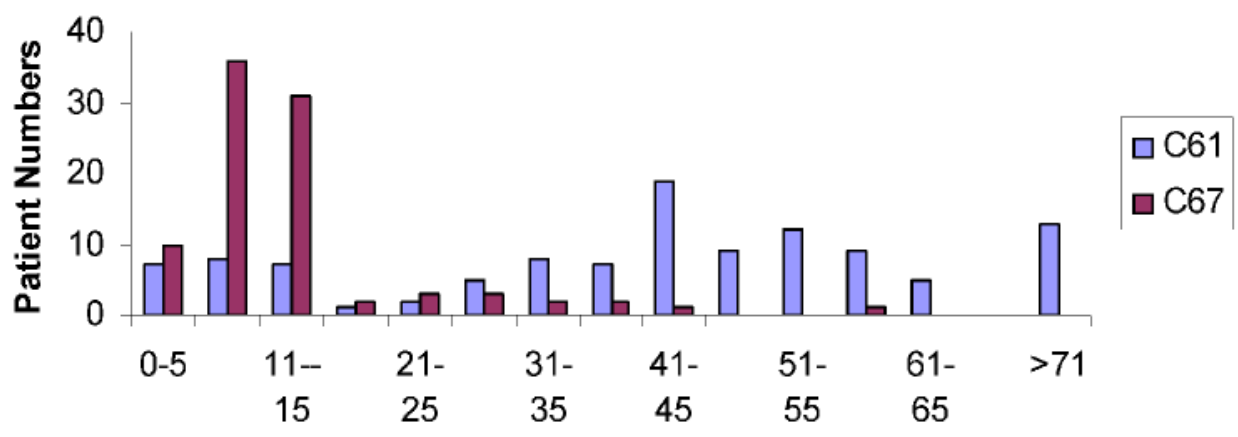

Days to Decision to Treat

Figure 1 Average waiting times for Bladder (C67) and Prostate (C61) patients

To understand which patients were in each category, we plotted patient numbers versus days to decision to treat for each urology cancer type, based on the International Classification of Diseases (ICD10) code. This immediately showed that the original sustainability graph was made up of two distinct components, one for patients with bladder cancer (C67) and one for prostate cancer (C61) (Figure 1).

Re-plotting the urology sustainability graph for both bladder and prostate patients demonstrated the difference in sustainability between these two pathways (Figure 2). The new urology sustainability graph implies that the bladder pathway is highly sustainable, while the prostate pathway has a low sustainability; the combined plot is retained so that effect of splitting the data into the two distinct pathways can be visualized.

This result led us to suspect that the sustainability graphs of other cancer sites were not reflecting their true state. The original sustainability graph for the gynaecology site suggested a pathway that was nearly sustainable. However, analysis of the distribution of patients in relation to their type of cancer and days until decision to treat produced a separation of ovarian and uterine cancers (Figure 3) similar to the bladder and prostate above.

Again it can easily be seen that two distinct cohorts of patients exist. Re-plotting the sustainability graph for gynaecology patients with respect to their cancer type

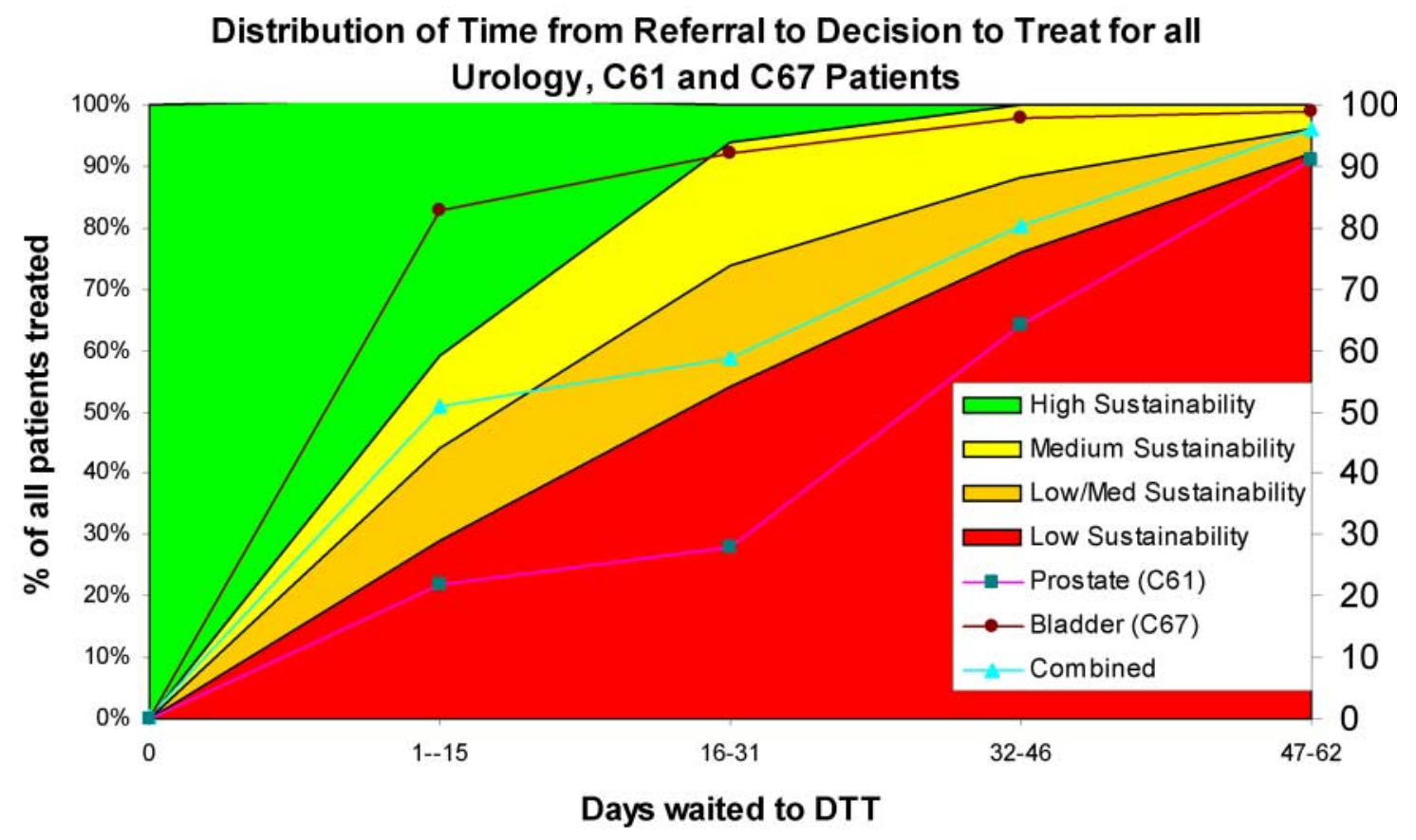




\section{Uterus (C54) and Ovary (C56)}

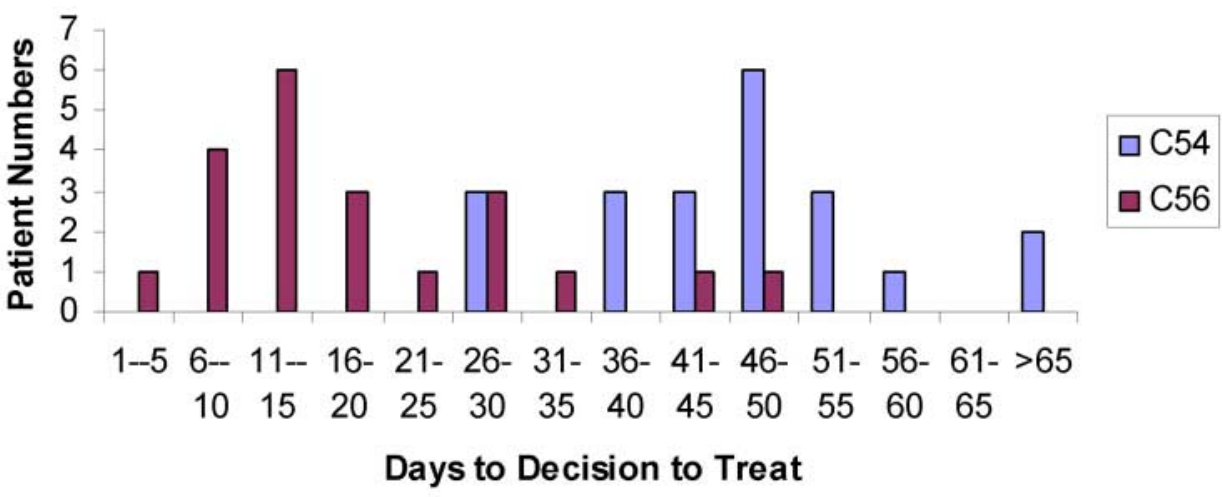

Figure 3 Average waiting times for Uterine (C54) and Ovarian (C56) patients

produced a graph that was far more informative. It is now evident that the pathway developed for ovarian cancer (C56) patients has medium sustainability, while the pathway for uterine cancer (C54) patients has low sustainability.

This result is also reflected in the average time to decision to treat for these patients. The average time to decision to treat for all gynaecology patients is 33 days; this breaks down into an average time to decision to treat of 45 days for uterine cancer (C54) patients and 18 days for ovarian cancer (C56) patients.

While the benefits of this approach can be demonstrated when pathways are distinctly different, pathways that are similar show no benefit. We saw this in the analysis of the upper-gastrointestinal cancer site. The initial sustainability graph showed a cancer site that is nearly sustainable, but further analysis (using the same type of bar chart as above) of the two main pathways within this site, namely oesophagus and stomach, showed that the average times to decision to treat were similar, although the spread of patients was different.

Cancer waiting times have been an important measure since specific targets were set in the NHS Cancer Plan $2000 .{ }^{1}$ Evidence has been produced that supports the view that the quicker a patient is diagnosed with and therefore treated for cancer, the better their survival prospects become. $^{2}$ There is still uncertainty about the optimum time patients should wait and whether this can be arbitrarily imposed for all cancer sites. On the other hand, patients

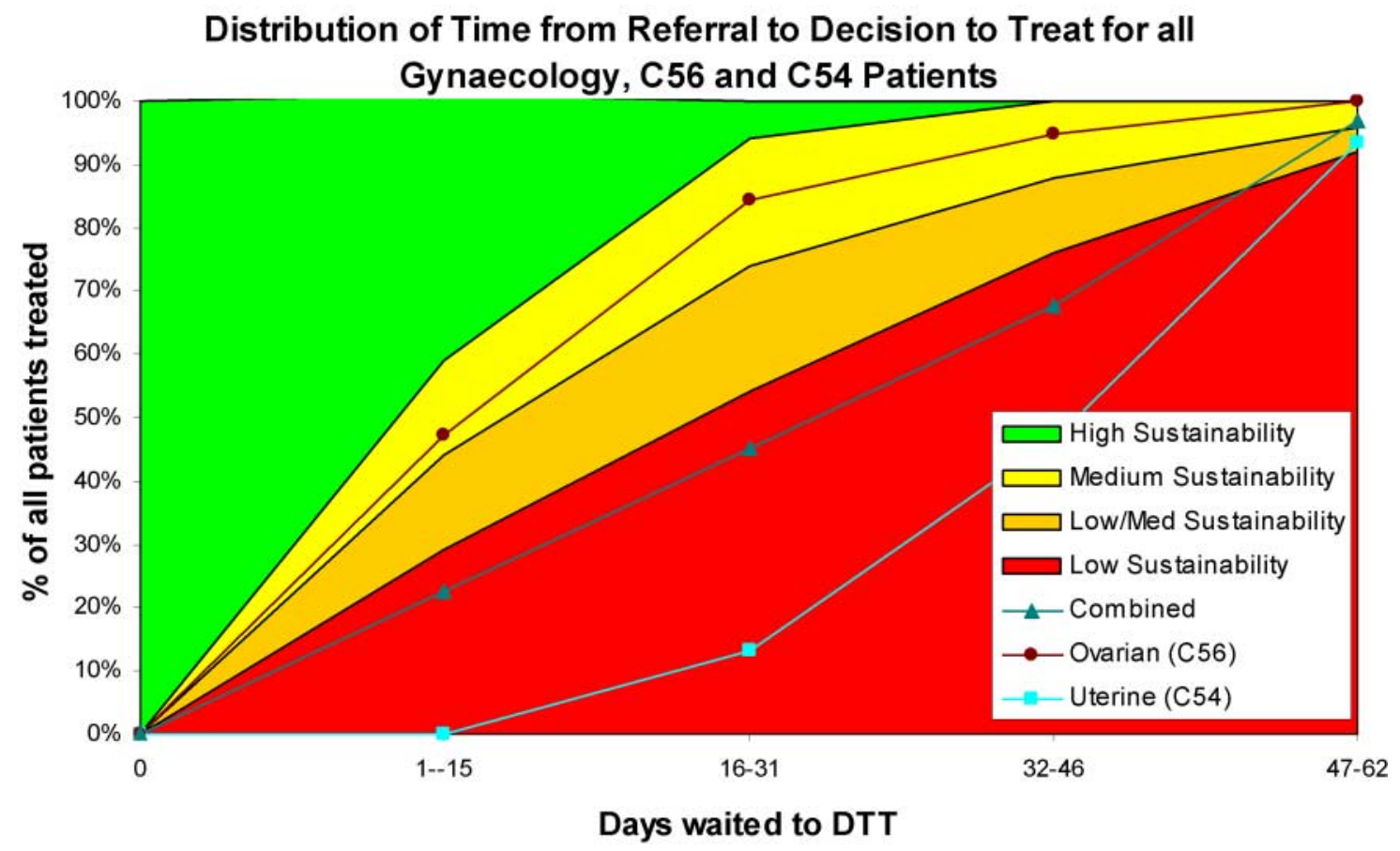

Figure 4 Sustainability graph for Uterine (C54), Ovarian (C56) and all Gynaecology patients 
and clinicians would not question that cancer should be treated in a timely fashion provided diagnostic and staging shortcuts are avoided.

The time to decision to treat for an individual patient can be used as an indication of whether treatment will be started within the 62-day period for fast track patients. It has been suggested that we should aim to make the 'decision to treat' by day 42 if the target is to be sustainable in the long term. Regardless of the actual time a patient should wait, the challenge for all cancer networks/acute trusts is to sustain the progress that has been made. To assist cancer networks in the analysis of their cancer sites, a sustainability tool has been produced that estimates the sustainability of a given pathway. This tool is heavily biased towards patients obtaining a decision to treat within 15 days. While such an approach is not a drawback it must be treated with a degree of caution.

The data we have presented above demonstrate that pathways can influence the sustainability rating for a given cancer site. In addition, the 42-day target for decision to treat would not lead to a highly sustainable rating for a pathway using the above measurements. Cancer patients present with a wide spectrum of symptoms. While in some cases these can be quickly and easily diagnosed and treatment given, especially if the diagnostic test is also considered the first treatment, other pathways are more complex and therefore protracted.

This paper links, for the first time, the time taken to decision to treat to the type of cancer diagnosed. It does not, however, go into a detailed study of why some cancer types can have a protracted diagnostic phase. The urology sustainability graphs described above ultimately reflect the fact that bladder cancer presents with a frightening symptom of blood in the urine and patients are referred to 'one-stop' haematuria clinics. The diagnostic cystoscopy is often the first treatment. Contrast this with prostate cancer, which presents with the nebulous symptoms of 'prostatism' - something every man experiences to varying degrees. If cancer is suspected on clinical grounds or because of a raised prostate-specific antigen (PSA) test, the diagnostic pathway is complex, including transrectal ultrasound and biopsy followed by computed tomography (CT) scan and bone scan if the biopsy confirms cancer. Only then can an informed decision to treat be made, and with the multiple available treatments patients often need time to consider their choice. Therefore it would seem misplaced to apply the same criteria to prostate cancer patients as could be applied to bladder cancer patients in terms of the time they should take to reach decision to treat.

The argument is similar for gynaecological cancers. Ovarian cancer often presents urgently, but unfortunately at an advanced stage, with pain and an abdominal or pelvic might be malignant and simultaneously stages it. The diagnosis is confirmed at surgery, which is also the first treatment. On the other hand, uterine cancer presents with mid cycle or postmenopausal bleeding and most patients with these symptoms do not have cancer. The diagnosis is made at hysteroscopy and biopsy, although if results are equivocal this may need to be repeated. Only in the minority of cancer cases are staging tests, including CT and Magnetic Resonance Imaging (MRI), justified. Therefore, the patient's journey for these two cancers within the same 'tumour site' varies considerably.

It is interesting, but perfectly understandable, that within upper gastrointestinal cancers the time to decision to treat is similar for oesophageal and gastric cancers. Both are diagnosed at first test, namely gastroscopy, but then ensues a complex staging pathway to identify the few patients who might benefit from high risk surgery. As a result, the time to decision to treat is protracted.

When investigating the sustainability of cancer pathways using this tool, health care workers should ensure that they are focusing on pathways that are specific. Such an approach should then enable them to estimate the efficiency of the given pathway and any beneficial changes should produce a move from low to high sustainability on the graph.

Reducing cancer waiting times is a commendable aim but this should not be achieved at the expense of appropriate staging and considered decision making. Considerable progress has been made in the NHS in reducing waiting times and streamlining patient pathways over the last few years. It is now important to work towards making these improvements sustainable and avoid short term gains.

With the above caveats, measuring sustainability with this tool will be useful in assessing the beneficial changes that can be made to individual cancer pathways. It will also indicate those pathways that require investigation, which should lead to a more streamlined and sustainable process. It should be emphasized that different cancer types, based on their ICD10 code, within each 'tumour site' would be expected to have different degrees of sustainability as measured by this tool. Breaking the sites down into their individual ICD10 cancer types will help to make this tool more useful.

We believe that a move away from the 'one size fits all' approach of cancer waiting times to one that recognizes the difficulties inherent in individual cancer pathways will be beneficial for future sustainability, as it will maintain buy-in from both clinicians and management. This will then lead to targets / standards which are based on realistic patient data rather than arbitrary numbers. It has previously been noted (JRSM 2006:99;10-13) that ' . . . poorly conceptualized targets deliver poor or perverse outcomes as an unintended consequence.' 7 
Competing interests None declared.

Funding This study was fully supported by the Cancer Services Collaborative 'Improvement Partnership'.

Ethical approval Not required.

Guarantor CS.

Contributorship RT had the initial idea for this article based on data produced by CS, which was later refined by both authors. CS wrote the initial draft and RT added the medical perspective. Both authors revised the manuscript critically and gave final approval of the version published.

\section{REFERENCES}

1 The NHS Cancer Plan: A Plan for Investment, A Plan for Reform. London: Department of Health, 2000
2 Richards MA, Westcombe AM, Love SB, Littlejohns P, Ramirez AJ. Influence of delay on survival in patients with breast cancer; a systematic review. Lancet 1999;353:1119-26

3 Richards M. Sustaining Cancer Waiting Times Standards. London: Department of Health, 2006

4 Cancer Service Collaborative 'Improvement Partnership'. Chapted 10: Sustaining Cancer Waiting Times through Effective Pathway Management. 'The How to Guide' Achieving Cancer Waiting Times. London: CSCIP, 2006

5 Richards M. Cancer Waiting Times: Action Required. London: Cancer Action Team, 2006

6 The bespoke software programme was written in the Microsoft's Visual Basic programming language. For each patient the programme will compute the time in days between each appointment attended, downloaded from the CWT data base 'Open Exeter'. These values are then summed to give a time from referral to decision to treat. This data is then used to generate the accompanying graphs. The programme will also extract the initial treatment and diagnosis. Further information and availability concerning this programme can be obtained from CS.

7 Lewis R, Appleby J. Can the English NHS meet the 18-week waiting list target? J R Soc Med 2006:99;10-3

\section{WIN THE PUBLICATION GAME}

\section{Kamran Abbasi and the Young Fellows of the Royal Society of Medicine invite you to learn how to improve your chances of getting published...}

'The most useful day I have had in years'

Lalitha D'Souza, Delegate, 3 June 2006

Run by Dr Kamran Abbasi, editor of the Journal of the Royal Society of Medicine, this is an extremely useful day for any trainee. Recently described as a triumph by the Lancet

(15 April 2006), this course is the ideal guide to the world of medical publishing.

Book now for October 2007

For further information or to book online, visit the Young Fellows

Committee page of the RSM website at ww.rsm.ac.uk/yf/

Alternatively, contact the Young Fellows at: tel (+44) 0207290 3846;

fax (+44) 0207290 2989; email young.fellows@rsm.ac.uk 